M. Stöhr, C. M. Arndt and W. Meier, Effects of Damköhler number on vortexflame interaction in a gas turbine model combustor, Proceedings of the Combustion Institute 34 (2013) 3107-3115.

The original publication is available at www.elsevier.com

http://dx.doi.org/10.1016/j.proci.2012.06.086 


\title{
Effects of Damköhler number on vortex-flame interaction in a gas turbine model combustor
}

\author{
M. Stöhr ${ }^{\mathrm{a}, *}$, C. M. Arndt ${ }^{\mathrm{a}}$, W. Meier ${ }^{\mathrm{a}}$ \\ ${ }^{a}$ German Aerospace Center (DLR), Institute of Combustion Technology, Pfaffenwaldring 38-40, 70569 Stuttgart, Germany
}

\begin{abstract}
The mechanisms of the interaction between a precessing vortex core (PVC) and a turbulent swirl flame in a gas turbine model combustor are investigated experimentally using simultaneous PIV and OH-PLIF at repetition rates up to $10 \mathrm{kHz}$. Three operating conditions with different flow rates corresponding to Damköhler numbers of $1,0.5$ and 0.3 are studied. The flames are mainly stabilized in the shear layer of the inner recirculation zone, where also the PVC is located. The measurements show that for all conditions, the PVC interacts strongly with the flame. Two main effects have been observed: On the one hand, the PVC leads to roll-up of the reaction zone at the flame base. This enhances the supply of heat and radicals to the unburned gas, and thus favors subsequent ignition of the unburned gas. On the other hand, the PVC causes considerable aerodynamic stretch of the reaction zones. This can lead locally to disruption, quenching or extinction of the flame. The strength of the two effects changes significantly when the Damköhler number changes. For the case $\mathrm{Da}=1$, the flame roll-up due to the PVC leads to a strong increase of heat release around the vortex. The stretch rates are relatively low and do not strongly affect the reaction progress. In the case $\mathrm{Da}=0.5$, the PVC initially also causes flame roll-up at the flame base. Subsequently, however, roll-up and propagation of the flame are affected by local disruptions and quenching of the reaction zone due to the increased vortex-induced aerodynamic stretch. Consequently, part of the heat release is delayed and shifted to locations further downstream. For $\mathrm{Da}=0.3$, aerodynamic stretch rates further increase and the reaction zones are even more affected by local disruptions and quenching. The zone of heat release is therefore even further elongated in the direction of the flow.
\end{abstract}

Keywords: Turbulent swirl flames, Precessing vortex core, Vortex-flame interaction, Gas turbine combustion, High-speed laser diagnostics

\section{Introduction}

Structure and stability of turbulent flames in gas turbine (GT) combustors are largely influenced by intricate interactions between flow and chemistry. Their exact mechanisms, however, are in many respects still not well enough understood [1,2], and further improvements of GT combustors with respect to emissions, fuel flexibility, and reliability therefore require additional studies of these interactions. Apart from turbulent fluctuations, the flow fields often contain unsteady large-scale coherent vortex structures that may also interact with the flame. For swirl burners, which are most often employed in GT combustors, a prominent example of such structures is the so-called precessing vortex core (PVC). While flow pattern and frequency of PVCs have been frequently characterized (literature surveys can be found in [3, 4]), only few studies of their interaction with a flame are available. The works of Syred and coworkers [5, 6, 7], Li and Gutmark [8] showed effects of the PVC on mixing and flame shape, and studies of Stöhr et al. [9, 4] found that the PVC causes increased heat release due to flame roll-up.

The named studies focused on PVC-flame interactions at one certain operating condition. The interaction of a vortex with a

${ }^{*}$ Corresponding author. Email: michael.stoehr@dlr.de

Preprint submitted to Proceedings of the Combustion Institute flame, however, generally depends on many parameters like, e.g., local gas composition and temperature, relative size and orientation of flame front and vortex, and time scales of flow and chemistry (see Ref. [10] for an extensive review). It is thus expected that the mechanism of the interaction changes, e.g., when the operating condition is changed, or when a scaled prototype is replaced by a full-scale burner. Understanding these changes is therefore a key issue for the design of improved GT combustors.

The present study investigates PVC-flame interactions in a GT model combustor for three operating conditions. The three conditions correspond to values of the Damköhler number Da (i.e., ratio of flow time scale and chemical time scale) of $1,0.5$ and 0.3. The analysis of the interactions is based on simultaneous measurements of flow field and flame structure using time-resolved PIV and OH-PLIF. The study continues two previous works on PVC-flame interaction in the same combustor: In Ref. [9], a method for estimating the phase angle of the PVC using a Proper Orthogonal Decomposition of the velocity fields was used to characterize the phase-averaged dynamics of flow field and flame structure. The method also allowed determination of the 3D structure of the PVC from 2D phase averages. In the other work [4], the PVC-flame interaction was charac-

September 20, 2012 


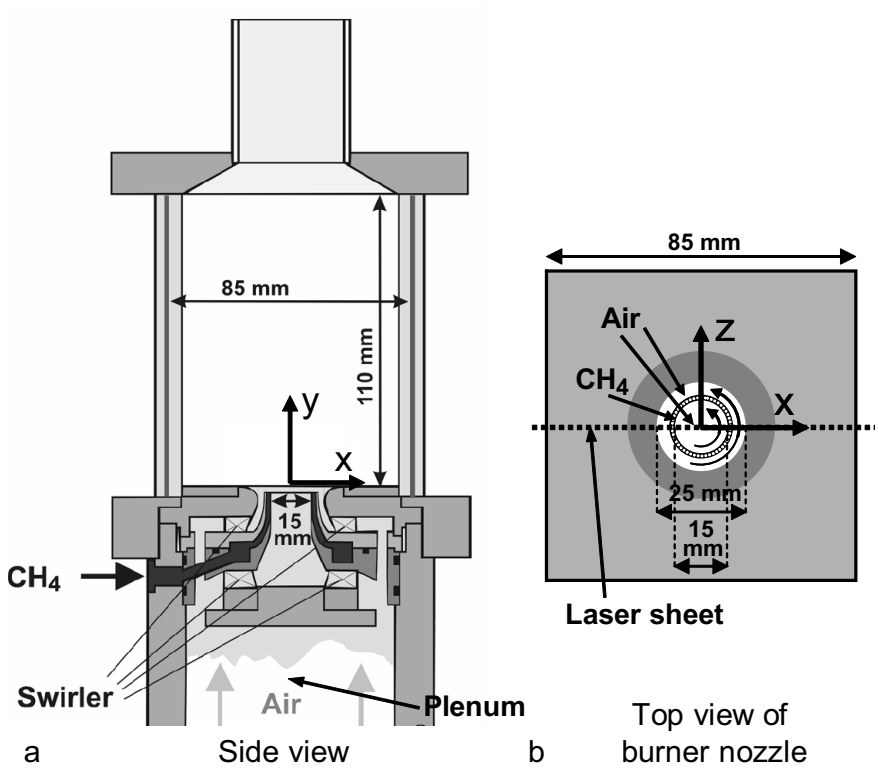

Figure 1: Sketch of gas turbine model combustor

terized in detail for the case $\mathrm{Da}=1$ using time-resolved PIV and OH-PLIF with a recording rate of $5 \mathrm{kHz}$. The cases $\mathrm{Da}=0.5$ and $\mathrm{Da}=0.3$, which are adressed in the present study, imply significantly higher flow velocities. In order to maintain an appropriate temporal resolution, the experimental setup was extended so that it now provides a recording rate of $10 \mathrm{kHz}$.

\section{Experimental setup}

\subsection{Combustor and operating conditions}

A schematic of the gas turbine model combustor is shown in Fig. 1. The air first enters a plenum (diameter $79 \mathrm{~mm}$, height $65 \mathrm{~mm}$ ) and then separately passes two radial swirl generators. The two co-swirling flows enter the combustion chamber through a central nozzle (diameter $15 \mathrm{~mm}$ ) and an annular nozzle (inner diameter $17 \mathrm{~mm}$, outer diameter $25 \mathrm{~mm}$ contoured to an outer diameter of $40 \mathrm{~mm}$ ). Non-swirling $\mathrm{CH}_{4}$ is fed through 72 channels $\left(0.5 \times 0.5 \mathrm{~mm}^{2}\right)$ forming a ring between the air nozzles. The exit planes of the fuel and central air nozzles are located $4.5 \mathrm{~mm}$ below the exit plane of the outer air nozzle which is taken as height $y=0 \mathrm{~mm}$. The combustion chamber has a square cross-section of $85 \times 85 \mathrm{~mm}^{2}$, a height of 114 $\mathrm{mm}$, and is enclosed by four quartz windows allowing optical access for lasers and cameras. A conical top plate with a central exhaust tube (diameter $40 \mathrm{~mm}$, length $50 \mathrm{~mm}$ ) forms the exit.

Three operating conditions with a global equivalence ratio $\phi=0.65$ and thermal powers of $\mathrm{P}=10,20$ and $35 \mathrm{~kW}$ are studied (see Table 1). In all cases, the flame is operated with methane and dry air at room temperature and atmospheric pressure. The Reynolds number of the unburned inflow ranges from $\mathrm{Re}=15000$ at $P=10 \mathrm{~kW}$ to $\mathrm{Re}=52500$ at $P=35 \mathrm{~kW}$. From velocity profiles at $y=1 \mathrm{~mm}$, the swirl number $S$ has been estimated in a previous work as $S=0.55$ at $P=10 \mathrm{~kW}$ and $S=0.9$ at $P=35$
Flame 1 Flame 2 Flame 3

\begin{tabular}{lccc}
\hline \hline$P[\mathrm{~kW}]$ & 10 & 20 & 35 \\
\hline $\mathrm{Re}$ & 15000 & 30000 & 52500 \\
\hline$f_{\mathrm{PVC}}[\mathrm{Hz}]$ & 500 & 1000 & 1750 \\
\hline$u(R)[\mathrm{m} / \mathrm{s}]$ & 8 & 17 & 28 \\
\hline $\mathrm{Da}$ & 1 & 0.5 & 0.3
\end{tabular}

Table 1: Flame parameters

$\mathrm{kW}$ [11]. Additional parameters of the flames with respect to vortex-flame interaction are discussed in Sect. 4.

\subsection{Measuring techniques}

The main part of the measurements was performed with a combined stereo-PIV and OH-PLIF technique, which was used in two configurations: For the condition with $P=10 \mathrm{~kW}$, a system with a repetition rate of $5 \mathrm{kHz}$ was employed, whereas for $P=20$ and $35 \mathrm{~kW}$, a version with $10 \mathrm{kHz}$ was used (which is the maximum practicable rate for the present system). Below the 5 $\mathrm{kHz}$ system is briefly described (further details are provided in Ref. [12]), and then additional specifications are given for the system with $10 \mathrm{kHz}$.

The $5 \mathrm{kHz}$ stereo-PIV system is based on a dual-cavity Nd:YAG laser (Edgewave IS-6IIDE, $2.6 \mathrm{~mJ} /$ pulse at $532 \mathrm{~nm}$ ) and a pair of CMOS cameras (LaVision HSS 5). The OH-PLIF system consists of a dye laser (Sirah Cobra-Stretch HRR, 100 $\mu \mathrm{J} /$ pulse at $283.2 \mathrm{~nm}$ ) pumped with a Nd:YLF laser (Edgewave IS-8IIE, $4 \mathrm{~mJ} /$ pulse at $523 \mathrm{~nm}$ ) and an intensified CMOS camera (LaVision HSS 6) equipped with a bandpass filter (300-325 $\mathrm{nm})$. The beams of the two laser systems are expanded into two coplanar vertical light sheets across the central section $(z=0$ $\mathrm{mm}$ ) of the combustion chamber (Fig. 1b). In the chamber, the thickness of the laser sheets was $0.7 \mathrm{~mm}$ and $0.4 \mathrm{~mm}$ for PIV and PLIF, respectively. While the field of view for the stereoPIV was limited to a $30 \times 30 \mathrm{~mm}^{2}$ region above the nozzle, the OH-PLIF field extends across the full width and up to a height of $y=45 \mathrm{~mm}$. All cameras had sufficient on-board memory for 4096 individual recordings corresponding to an acquisition time of $0.8 \mathrm{~s}$. For the OH-PLIF images, mean background flame luminosity was subtracted, and spatial non-uniformity of the laser sheet and imaging system response was corrected using a mean image derived from laser-induced fluorescence of acetone vapor in the combustion chamber.

At the time of the $10 \mathrm{kHz}$ measurements, additional hardware was available, and therefore the following changes were made compared to the $5 \mathrm{kHz}$ system. For the stereo-PIV, a combination of the LaVision cameras HSS 6 and HSS 8 was used, which provided a larger field of view of $40 \times 40 \mathrm{~mm}^{2}$. For OH-PLIF, a Sirah Credo dye laser $(165 \mu \mathrm{J} /$ pulse at $283.2 \mathrm{~nm})$ and an intensified LaVision HSS 5 camera were used. The on-board memory of the cameras was sufficient for 8192 individual recordings.

The in-plane spatial resolution of velocity is $1.5 \mathrm{~mm}$ for 5 $\mathrm{kHz}$ PIV and $3 \mathrm{~mm}$ for $10 \mathrm{kHz}$ PIV. Based on the \pm 0.1 pixel uncertainty of the cross-correlation peak-finding algorithm, the random uncertainty of the PIV measurements is estimated as 


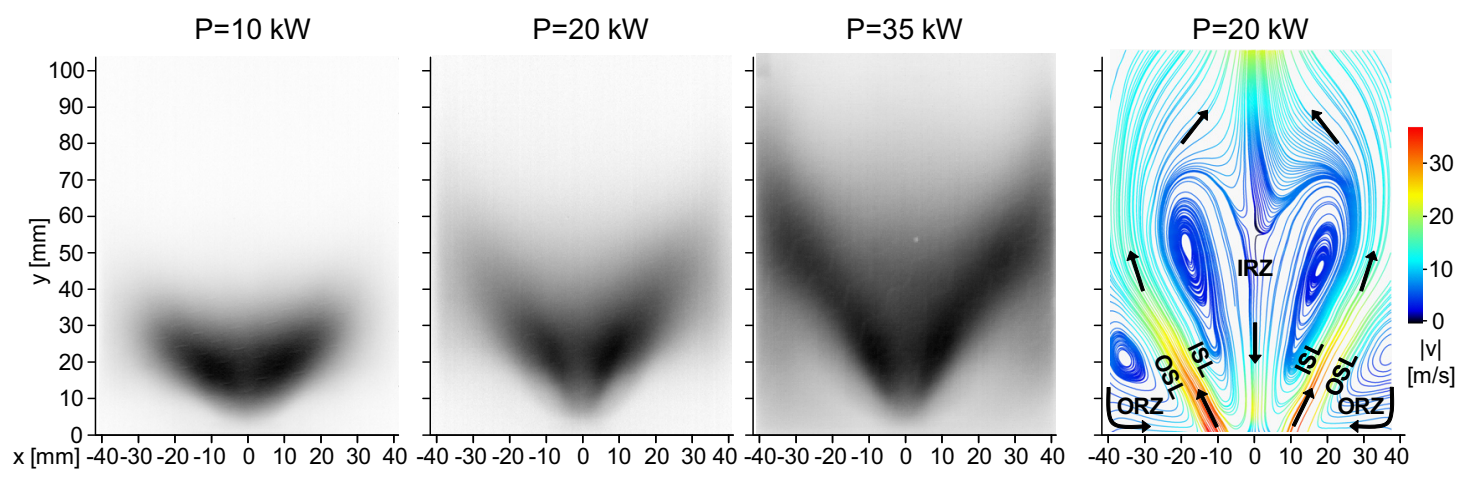

Figure 2: Average flame shapes for $P=10,20$ and $35 \mathrm{~kW}$, and average flow field for $P=20 \mathrm{~kW}$.

$\pm 0.5 \mathrm{~m} / \mathrm{s}, \pm 0.8 \mathrm{~m} / \mathrm{s}$ and $\pm 1.2 \mathrm{~m} / \mathrm{s}$ for flames 1,2 and 3 , respectively.

Image sequences of $\mathrm{OH}$ chemiluminescence were recorded using the OH-PLIF cameras with an exposure time of $40 \mu \mathrm{s}$.

\section{Flame shapes and flow fields}

\subsection{Averages of $\mathrm{OH}-\mathrm{CL}$ and velocity}

Figure 2 shows average $\mathrm{OH}$ chemiluminescence (CL) images of the three flames. The OH-CL signal (integrated along the line-of-sight) is used as a marker of the locations of heat release [13]. At $P=10 \mathrm{~kW}$, heat release takes place in a conically shaped zone between $y=5 \mathrm{~mm}$ and $y=45 \mathrm{~mm}$ and within a radius of $r<35 \mathrm{~mm}$ from the central axis. When thermal power and thus flow velocities are increased, the upper end of the flame conus moves up to $y=80 \mathrm{~mm}$ for $P=35 \mathrm{~kW}$. The flame base, by contrast, remains constant at a height of $y \approx 5 \mathrm{~mm}$. Raman measurements of major species concentrations have shown that strong mixing occurs between the fuel nozzle exit ( $y=-4.5 \mathrm{~mm}$ ) and the flame base, such that the flames have a largely premixed nature. For the case $P=35 \mathrm{~kW}$, further detailed informations about the thermochemical state can be found in Refs. [11, 14].

The average flow field is shown only for $P=20 \mathrm{~kW}$ since its structure for the other two flames is almost identical (cf. Ref. [4]). The structure of the flow is typical of confined swirl flames. Unburned gas enters the chamber through the nozzle at the bottom in the form of a cone-shaped stream. Part of the burned gas flows back towards the nozzle through the inner (IRZ) and outer (ORZ) recirculation zones. This provides a source of heat and radicals to the unburned gas, which enhances ignition and thus facilitates flame stabilization. Strong velocity gradients occur in the inner shear layer (ISL) between the inflow and the IRZ, and in the outer shear layer (OSL) between inflow and ORZ.

\subsection{Precessing vortex core}

Figure 3 shows an instantaneous velocity field for $P=20 \mathrm{~kW}$. The most distinctive feature compared to the average flow is the zig-zag arrangement of vortices in the ISL marked by black arrows. This arrangement indicates the presence of a coherent 3D helical vortex in the ISL. This helical vortex is found

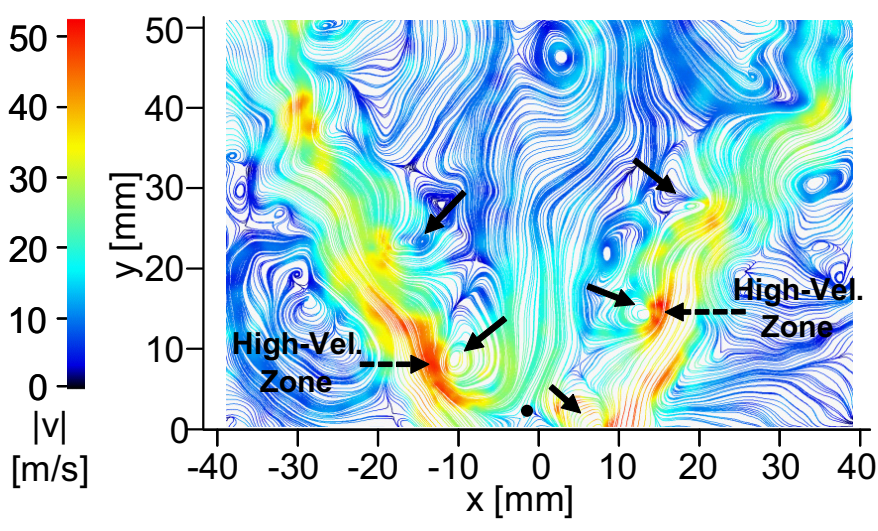

Figure 3: Instantaneous velocity field for $P=20 \mathrm{~kW}$.

in many GT-typical swirl flames (a literature survey is provided in Ref. [4]) and is commonly termed a precessing vortex core (PVC). It rotates around the central axis $(x=z=0 \mathrm{~mm})$ with a frequency $f_{\mathrm{PVC}}$ that scales linearly with the flow rate. The values of $f_{\mathrm{PVC}}$, which were obtained from the time-resolved PIV measurements, are specified in Table 1.

Various features of the flow field are linked to the PVC, such as the unsteady lower stagnation point (marked with a black dot), where hot burned gas and fresh gas collide frontally. A recent work [4] has shown that this point (which is not present in the average flow field, cf. Fig. 2) plays an important role in the stabilization of the flames. Another feature that is particularly relevant for the present work are the zones of high velocity near the vortex centers, which are marked in Fig. 3. It will be seen in Sect. 5 that these zones induce large extensive strain rates and thereby considerably influence the flame dynamics. Further characteristics of the PVC can be found in Ref. [4].

\section{Parameters of vortex-flame interaction}

\subsection{Damköhler number}

In a reacting flow, the interaction of flow and chemistry is commonly characterized by the Damköhler number $\mathrm{Da}=\tau_{f} / \tau_{c}$. 


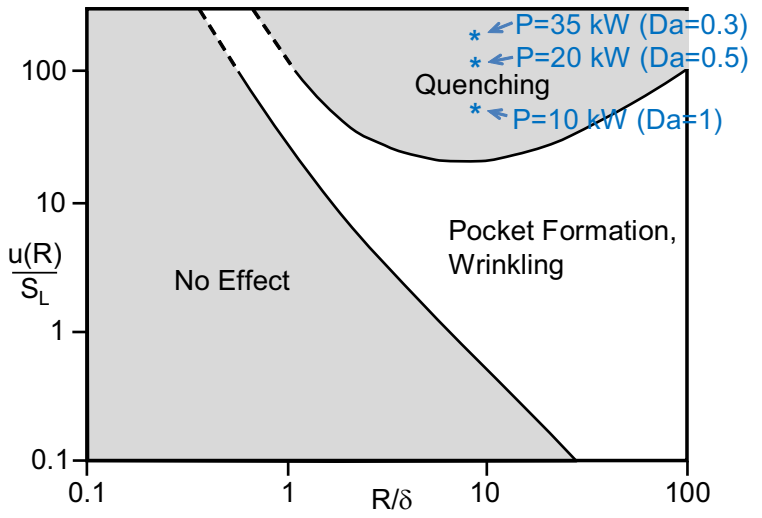

Figure 4: Spectral diagram of vortex-flame interaction (adapted from Ref. [18])

$\tau_{f}$ and $\tau_{c}$, respectively, denote the time scales of flow and chemistry. The determination of $\tau_{f}$ and $\tau_{c}$, however, is somewhat arbitrary since both the flow field and chemical reactions cover a certain range of time scales (for a detailed discussion of this issue see, e.g., Ref. [15]).

For the present study of the PVC, the time scale of the flow is chosen as the period of the PVC precession, $\tau_{f}=1 / f_{\mathrm{PVC}}$. The chemical time scale is chosen as the ratio of the reaction zone thickness $\delta$ and the laminar flame speed $S_{\mathrm{L}}$, i.e., $\tau_{c}=\delta / S_{\mathrm{L}}$. For methane and air at $\phi=0.65$ and atmospheric pressure, the value of $S_{\mathrm{L}}$ is $\approx 0.16 \mathrm{~m} / \mathrm{s}$ [16]. Using the present combustor at conditions similar to those studied in this work, the reaction zone thickness was estimated in a previous study by means of $\mathrm{CH}$ PLIF as $\delta \approx 0.35 \mathrm{~mm}$ [17]. Accordingly, the value of $\tau_{c}$ is $\approx 2$ ms. The resulting values of $\mathrm{Da}$ range from $\mathrm{Da}=1$ at $P=10 \mathrm{~kW}$ to $\mathrm{Da}=0.3$ at $P=35 \mathrm{~kW}$ as specified in Table 1 . From the uncertainties of $S_{\mathrm{L}}, \delta$ and $f_{\mathrm{PVC}}$, the uncertainties of Da are estimated as $\pm 20 \%$.

\subsection{Spectral diagram}

For the particular case of vortices interacting with a flame, a more specific characterization is provided by so-called spectral diagrams [15]. These diagrams are based on two dimensionless parameters. The first is the ratio of the vortex radius $R$ and $\delta$, and the second is the ratio of the vortex velocity $u(R)$ and $S_{\mathrm{L}}$. Using DNS of turbulent premixed flames, Poinsot et al. [18] identified different regimes of vortex-flame interaction that correspond to certain regions in the spectral diagram. As shown in Fig. 4, the vortices have no effect on the flame when the ratios are relatively low, whereas for higher values wrinkling, pocket formation or quenching may occur.

For the present study, the values of $R$ and $u(R)$ are determined from 3D representations of the flow field of the PVC that were obtained in a recent work [4]. It was found that, when moving radially outward from the helical centerline of the PVC, the velocity first increases linearly until it reaches a maximum and then decays non-linearly. $R$ and $u(R)$ are thus chosen as the radius and the velocity at that maximum. For all three operating conditions, the value of $R$ in the flame zone is about $R=3 \mathrm{~mm}$.
The according values of $u(R)$ are given in Table 1 . The resulting locations in the spectral diagram are marked in Fig. 4 and indicate that all three conditions are located in the quenching regime. It is thus expected that, to a greater or lesser extent, the PVC causes local weakening or extinction of the reaction.

\subsection{Flame stretch}

The effect of a vortex on a flame mainly results from aerodynamic flame stretch $[19,20,21]$. Generally the flame stretch rate $\kappa$ is given by the relative change of the flame surface $A$, i.e., $\kappa=(1 / A) d A / d t$. If velocities and flame structure are measured in a two-dimensional plane (e.g., using PIV and OH-PLIF), the measurable part of the stretch rate is given by $[22,20]$

$$
\kappa=\kappa_{s}+\kappa_{c}=\partial u_{T} / \partial s-S_{\mathrm{L}} / r .
$$

Here $u_{T}$ is the velocity component that is tangential to the flame surface, $s$ denotes the coordinate along the flame surface, and $r$ is the local radius of curvature of the flame. The first term $\kappa_{s}$ is the aerodynamic flame stretch, and the second term $\kappa_{c}$ is the surface variation due to flame curvature.

The maximum stretch rate that a steady flame can withstand before extinction occurs is the extinction stretch rate $\kappa_{\text {ext }}$. For a premixed opposed jet flame operated with methane and air, Ju et al. [23] determined the extinction stretch rate for $\phi=0.65$ as $\kappa_{\text {ext }}=1000 \mathrm{~s}^{-1}$. This value of $\kappa_{\text {ext }}$, however, is limited to steady conditions. Several studies have found that for short time periods, flames can withstand much higher stretch rates [24, 25, 26]. The extinction limits under unsteady conditions, however, can hardly be quantified as they are also strongly history dependent.

\section{Mechanisms of vortex-flame interaction}

\subsection{General remarks}

In Sect. 3.1 (Fig. 2) it was seen that as the thermal power increases (Da reduces), the flame becomes elongated in the direction of the flow. In principle, this elongation is expected since the chemical time scales remain similar while the flow velocity increases. In this section, the underlying mechanisms of vortexflame interaction that are related to this change of flame shape will be investigated. For each condition, a time-resolved measurement of velocity field and flame structure will be discussed. Finally, a phase-averaged representation will be provided.

\section{2. $D a=1(P=10 \mathrm{~kW})$}

For the case $\mathrm{Da}=1$, Fig. 5 shows a time-series of velocity fields and flame structures obtained with simultaneous stereoPIV and OH-PLIF. The velocity fields reveal zig-zag ordered vortices representing the PVC, which are marked with dashed arrows at $t=0 \mathrm{~ms}$. The observable downstream motion of the vortices in the $2 \mathrm{D}$ images corresponds to the rotation of the $3 \mathrm{D}$ helical vortex around the central axis. At $t=0.6 \mathrm{~ms}$, another vortex appears on the left-hand side of the nozzle and subsequently propagates downstream.

The grayscale OH-PLIF images show the corresponding dynamics of the flame. In the OH-PLIF images, regions without $\mathrm{OH}$ (black) represent gas with low or medium temperature $(T<1500 \mathrm{~K})$, i.e., unburned gas with possible admixture of 

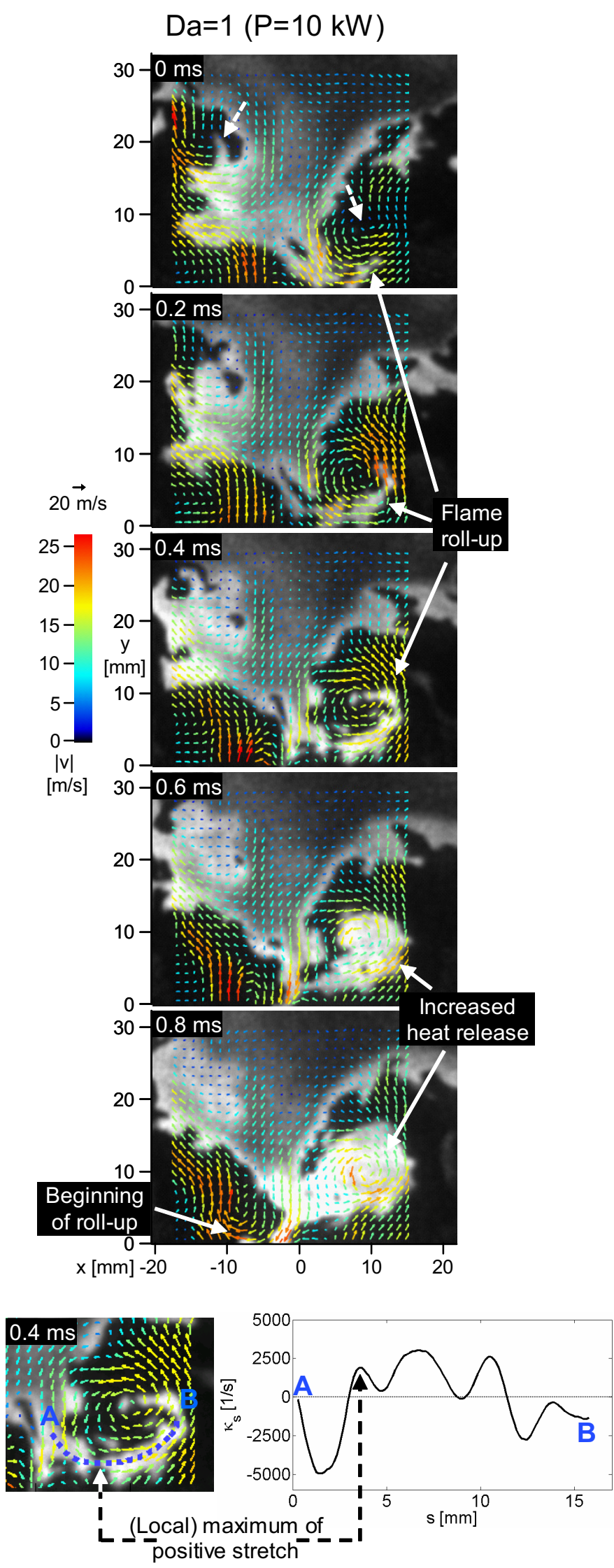

Figure 5: Time-series of PIV and OH-PLIF measurements for Da=1 burned gas. High levels of $\mathrm{OH}$ (light gray to white) indicate superequilibrium $\mathrm{OH}$, which is formed in the reaction zones and has a half-life period of $\approx 1 \mathrm{~ms}$ [27]. Regions with medium and low levels of $\mathrm{OH}$ (medium and dark gray) represent burned gas whose $\mathrm{OH}$ concentration has decayed toward equilibrium while it was transported away from the reaction zone.

At $t=0 \mathrm{~ms}$, part of the burned gas from the IRZ is deflected from the lower stagnation point to the right side. In the following images ( $t=0.2 \mathrm{~ms}$ and $t=0.4 \mathrm{~ms}$ ), the deflected burned gas is rolled up by the vortex on the right-hand side. The roll-up enlarges the flame surface, which increases the supply of heat and radicals to the adjacent unburned gas and thereby promotes ignition of the unburned gas. At $t=0.6 \mathrm{~ms}$, the $\mathrm{OH}$ levels in the region around the vortex have increased significantly, which indicates high rates of heat release. These high rates continue at $t=0.8 \mathrm{~ms}$, while the vortex that appears at $t=0.6 \mathrm{~ms}$ starts to roll-up the reaction zone on the left-hand side of the nozzle. It thereby initiates a similar dynamics as that seen on the righthand side before. In the following frames (not shown), similar vortex-induced dynamics of the flame periodically take place on the left and right side of the ISL.

The lower part of Fig. 5 provides an analysis of the stretch rate $\kappa$ that is exerted by the vortex on the flame at $t=0.4 \mathrm{~ms}$. The contribution of curvature-induced stretch $\kappa_{c}$ was estimated to be negligible $\left(\kappa_{c}=32 \mathrm{~s}^{-1}\right.$ for $r=5 \mathrm{~mm}$ and $\left.S_{\mathrm{L}}=0.16 \mathrm{~m} / \mathrm{s}\right)$. The aerodynamic stretch rate $\kappa_{s}$ was determined as described in Sect. 4.3 along the curved reaction zone (marked by the dotted blue line). It is found that along the reaction zone, the stretch rate oscillates between $\kappa_{s}=-5000 \mathrm{~s}^{-1}$ and $\kappa_{s}=3000 \mathrm{~s}^{-1}$. The highest values are therefore well above the (steady) extinction stretch rate $\kappa_{\mathrm{ext}}=1000 \mathrm{~s}^{-1}$. At one location $(s=4 \mathrm{~mm}$ ), local quenching of the reaction appears, which coincides with a local maximum of the stretch rate of $\kappa_{s} \approx 2000 \mathrm{~s}^{-1}$. The major part of the reaction zone, however, largely withstands the high stretch rates. This might be explained by the short duration that the high rates locally apply, by variability of local equivalence ratio, or by effects of out-of-plane motion.

\section{3. $D a=0.5(P=20 \mathrm{~kW})$}

An exemplary interaction of PVC and flame for $\mathrm{Da}=0.5$ is shown in the time-series in Fig. 6. The vortices representing the $\mathrm{PVC}$ at $t=0 \mathrm{~ms}$ are marked with dashed arrows. Similar to the case $\mathrm{Da}=1$, the vortex on the lower right-hand side first starts to roll up the reaction zone ( $t=0$ and $0.1 \mathrm{~ms}$ ). In contrast to $\mathrm{Da}=1$, however, the curved reaction zone subsequently becomes disrupted from the reactions in the IRZ ( $t=0.2 \mathrm{~ms})$. Apparently this is caused by the flame stretch induced by the high-velocity zone near the vortex center, as discussed in more detail in the following paragraph. After the disruption $(t=0.3$ and $0.4 \mathrm{~ms})$, the detached reaction zone moves downstream following the vortex. Unlike the situation for $\mathrm{Da}=1$, however, in this phase no significant increase of heat release is observed in the region around the vortex.

The stretch rates that occur during the disruption at $t=0.2 \mathrm{~ms}$ are shown at the bottom of Fig. 6. As for the case $\mathrm{Da}=1$, the aerodynamic stretch rate $\kappa_{s}$ was determined along the curved 

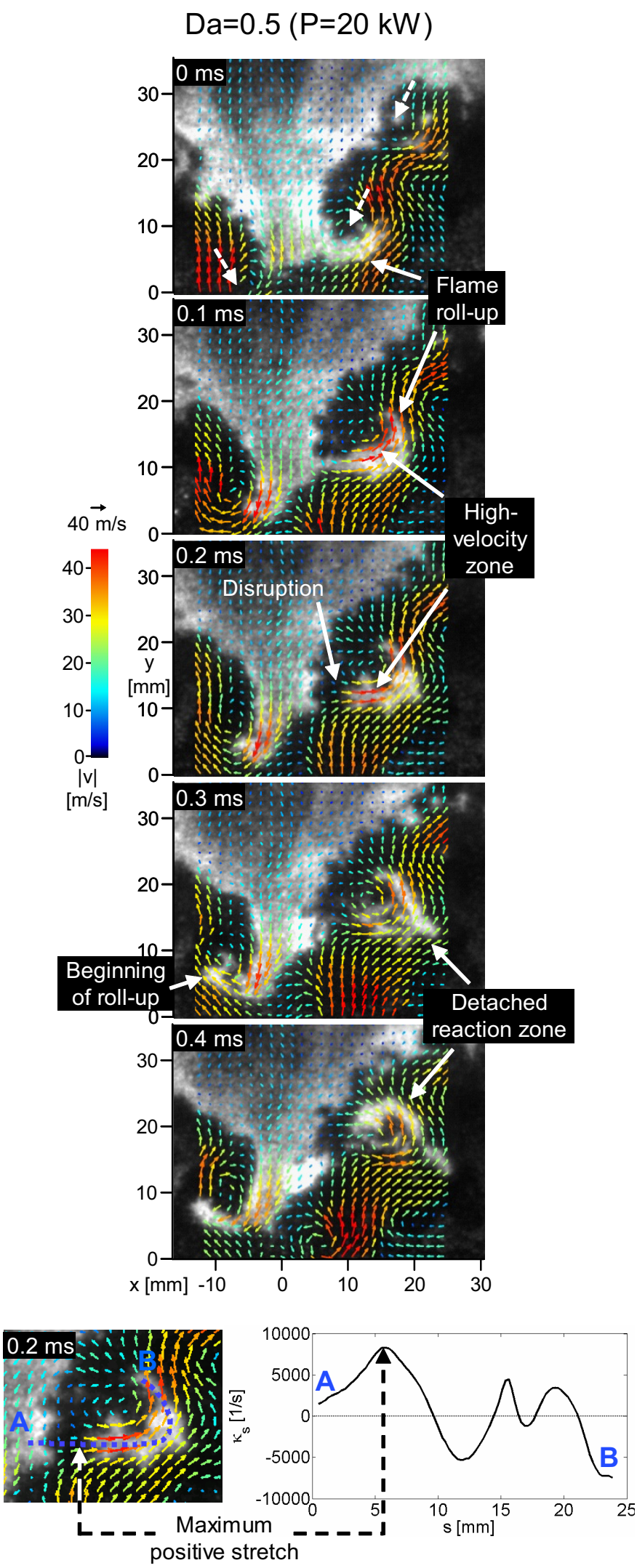

Figure 6: Time-series of PIV and OH-PLIF measurements for Da=0.5 zone marked by the dotted blue line. At the location of the disruption, the tangential velocity increases from $\approx 5 \mathrm{~m} / \mathrm{s}$ to $\approx 45$ $\mathrm{m} / \mathrm{s}$ over a distance of about $5 \mathrm{~mm}$. This induces a high positive stretch rate of $\kappa_{s} \approx 8000 \mathrm{~s}^{-1}$, which obviously causes the disruption. Additional peaks of the stretch rate are found at $s \approx 16$ and $19 \mathrm{~mm}$, which may be related to local reductions of $\mathrm{OH}$ appearing in the detached reaction zone at $\mathrm{t}=0.3 \mathrm{~ms}$.

In summary, the effect of the vortex on the flame has changed significantly compared to $\mathrm{Da}=1$. For $\mathrm{Da}=1$, the velocity gradients around the vortex induced an enlarged flame surface, but were not strong enough to cause significant quenching. At $\mathrm{Da}=0.5$, surface increase due to flame roll-up occurs only during an initial phase, whereas afterwards disruption and quenching occur due to the increased velocity gradients.

\section{4. $D a=0.3(P=35 \mathrm{~kW})$}

Figure 7 shows a time-series of vortex-flame interaction for $\mathrm{Da}=0.3$. At $t=0$ and $0.1 \mathrm{~ms}$, the vortex on the lower right side (dashed arrow) causes a deflection and slight roll-up of the reaction zone. Similar to $\mathrm{Da}=0.5$, the curved reaction zone subsequently becomes disrupted from the reactions in the $\operatorname{IRZ}(t=0.2$ $\mathrm{ms}$ ). After the disruption ( $t=0.3$ and $0.4 \mathrm{~ms}$ ), only small fragments of the reaction zone are visible near the vortex.

The stretch rates during the disruption at $t=0.2 \mathrm{~ms}$ are shown at the bottom of Fig. 7. Similar to the disruption at $\mathrm{Da}=0.5$, a local maximum of stretch rate $\left(\kappa_{s} \approx 7000 \mathrm{~s}^{-1}\right.$ at $\left.s \approx 4 \mathrm{~mm}\right)$ coincides with the point where the reaction zone disconnects. Furthermore, high stretch rates up to $\kappa_{s}=10000 \mathrm{~s}^{-1}$ appear at $s \approx 12$ $\mathrm{mm}$. This location corresponds to the detached part of the reaction zone. The high stretch rates may thus explain the additional fragmentation of the reaction zone at $t=0.3$ and $0.4 \mathrm{~ms}$.

The results show that the basic mechanism of vortex-flame interaction is similar for $\mathrm{Da}=0.3$ and $\mathrm{Da}=0.5$. For $\mathrm{Da}=0.3$, however, the effects of quenching and fragmentation of the reaction zones are significantly stronger due to the increased stretch rates.

\subsection{Phase averages}

The time-series discussed in Sect. 5.2-5.4 showed only one exemplary manifestation of vortex-flame interaction for each case. In the following, a phase-averaged analysis of the interactions is discussed, which is based on a large number of measurements (4096 and 8192 for $\mathrm{Da}=1$ and $\mathrm{Da}=0.3 \& 0.5$, respectively). The phase angle $\varphi$ is defined with respect to the periodic precession of the PVC. The value of $\varphi$ is determined for each individual flow field using a Proper Orthogonal Decomposition of the flow fields as described in Ref. [9]. For the corresponding OH-PLIF images, the structure of the reaction zone is determined using the gradient of $\mathrm{OH}$ and a subsequent threshold binarization. Phase averages of the reaction zone were then calculated for eight phase angles: $\varphi=0^{\circ}, 45^{\circ}$, $90^{\circ}, \ldots, 315^{\circ}$. At each of these phase angles, measurements in the range $\left[\varphi-22.5^{\circ} \ldots \varphi+22.5^{\circ}\right]$ have been averaged.

The results for $\varphi=0^{\circ}, 45^{\circ}, 90^{\circ}$ and $135^{\circ}$ are shown in Fig. 8 (the averages for $\varphi=180^{\circ}, 225^{\circ}, 270^{\circ}$ and $315^{\circ}$ are equivalent but mirrored at the central axis). For all three conditions, 

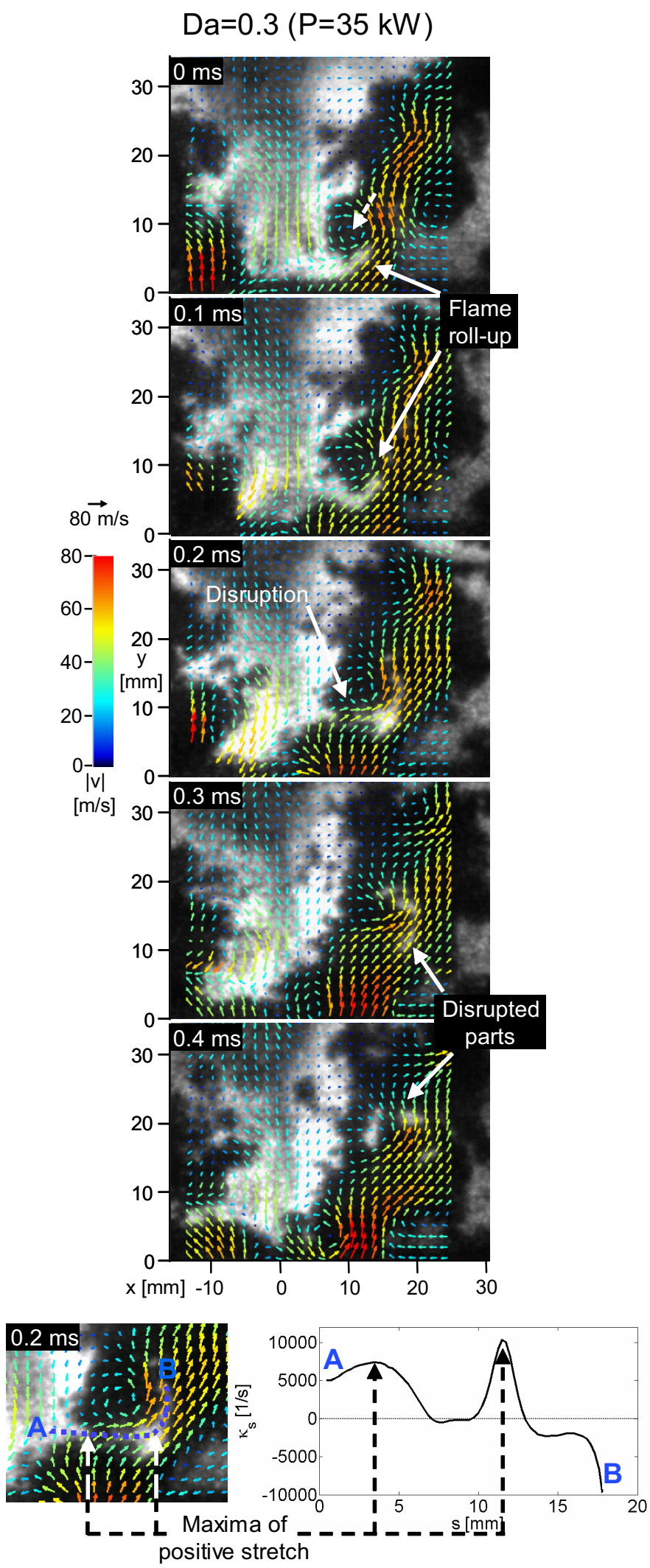

Figure 7: Time-series of PIV and OH-PLIF measurements for $\mathrm{Da}=0.3$ at $\varphi=0^{\circ}$ the reaction zone at the flame base is deflected to the right (the positions of the vortices at $\varphi=0^{\circ}$ roughly correspond to those at $t=0 \mathrm{~ms}$ in the respective time-series). The subsequent roll-up of the flame, however, differs considerably for the three conditions. The differences are highlighted in the region around the vortex marked with a square at $\varphi=90^{\circ}$. For $\mathrm{Da}=1$, intense roll-up and high heat release rates are seen in the vortex region. For $\mathrm{Da}=0.5$, flame roll-up takes place to a lesser extent, and the heat release is largely limited to the region near the IRZ (left side of square). For $\mathrm{Da}=0.3$, the roll-up and heat release near the vortex are reduced even further. Considering the results in Sects. 5.2-5.4, the decrease of reactions around the vortex at $\mathrm{Da}=0.5$ and 0.3 is evidently caused by disruptions and quenching due to increased flame stretch. The results demonstrate the importance of small-scale features such as the highvelocity zones near the vortices (see Fig. 3) to the large-scale structure of the flames.

\section{Summary and conclusions}

The mechanisms of the interaction between a precessing vortex core (PVC) and a turbulent swirl flame in a gas turbine model combustor have been investigated experimentally using simultaneous PIV and OH-PLIF at repetition rates up to 10 $\mathrm{kHz}$. Three operating conditions with different flow rates corresponding to Damköhler numbers of $1,0.5$ and 0.3 were studied. The flames are mainly stabilized in the shear layer of the inner recirculation zone, where also the PVC is located.

The results showed that for all conditions, the PVC interacts strongly with the flame. Two main effects have been observed: On the one hand, the PVC leads to roll-up of the reaction zone at the flame base. This enhances the supply of heat and radicals to the unburned gas, and thus favors subsequent ignition of the unburned gas. On the other hand, the PVC causes considerable aerodynamic stretch of the reaction zones. This can lead locally to disruption, quenching or extinction of the flame.

The strength of the two effects changes significantly when the Damköhler number changes. For the case $\mathrm{Da}=1$, the flame roll-up due to the PVC leads to a strong increase of heat release around the vortex. The stretch rates are relatively low and do not strongly affect the reaction progress. In the case $\mathrm{Da}=0.5$, the PVC initially also causes flame roll-up at the flame base. Subsequently, however, roll-up and propagation of the flame are affected by local disruptions and quenching of the reaction zone due to the increased vortex-induced aerodynamic stretch. Consequently, part of the heat release is delayed and shifted to locations further downstream. For $\mathrm{Da}=0.3$, aerodynamic stretch rates increase further and the reaction zones are even more affected by local disruptions and quenching. The zone of heat release is therefore elongated even further in the direction of the flow.

\section{References}

[1] R. S. Barlow, Proc. Combust. Inst. 31 (2007) 49-75.

[2] A. R. Masri, P. A. M. Kalt, Y. M. Al-Abdeli, R. S. Barlow, Combust. Theor. Model. 11 (2007) 653-673. 


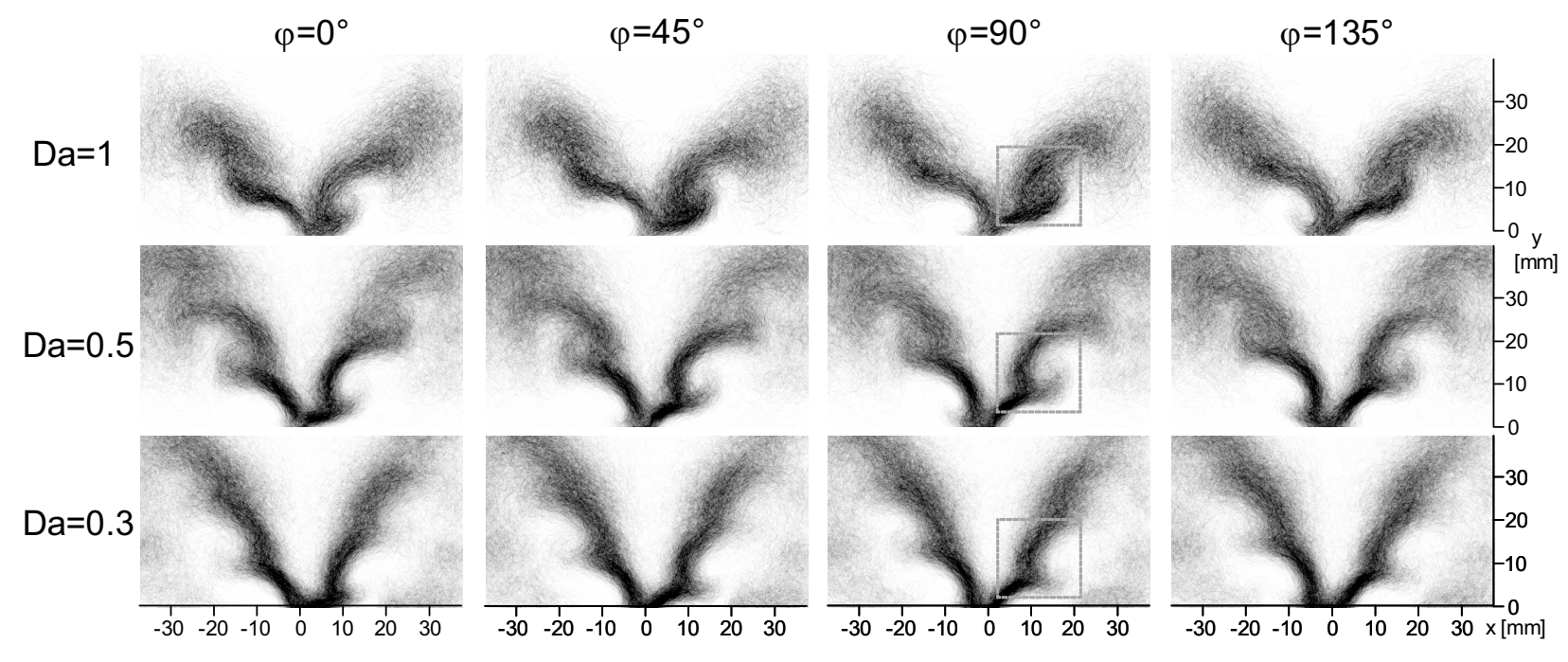

Figure 8: Phase averages of reaction zone.

[3] N. Syred, Prog. Energy. Combust. Sci. 32 (2006) 93-161.

[4] M. Stöhr, I. Boxx, C. D. Carter, R. Sadanandan, W. Meier, Combust. Flame 159 (2012) 26362649.

[5] N. Syred, J. M. Beér, Proc. Combust. Inst. 14 (1973) 537-550.

[6] N. Syred, A. K. Gupta, J. M. Beér, Proc. Combust. Inst. 15 (1975) $587-$ 597.

[7] N. Syred, W. Fick, T. O'Doherty, A. J. Griffiths, Combust. Sci. Technol. 125 (1997) 139-157.

[8] G. Li, E. J. Gutmark, 42nd AIAA Science Meeting and Exhibit (2004) AIAA-2004-0133.

[9] M. Stöhr, R. Sadanandan, W. Meier, Exp. Fluids 51 (2011) 1153-1167.

[10] P. H. Renard, D. Thévenin, J. C. Rolon, S. Candel, Prog. Energy Combust. Sci. 26 (2000) 225-282.

[11] P. Weigand, W. Meier, X. R. Duan, W. Stricker, M. Aigner, Combust. Flame 144 (2006) 205-224.

[12] I. Boxx, M. Stöhr, C. Carter, W. Meier, Combust. Flame 157 (2010) 15101525.

[13] Y. Hardalupas, M. Orain, Combust. Flame 139 (2004) 188-207.

[14] W. Meier, X.R. Duan, P. Weigand, Combust. Flame 144 (2006) 225-236.

[15] T. Poinsot, D. Veynante, Theoretical and Numerical Combustion, R. T. Edwards Inc., Philadelphia, USA, 2005

[16] F. N. Egolfopoulos, P. Cho, C. K. Law, Combust. Flame 76 (1989) $375-$ 391.

[17] W. Meier, X. R. Duan, P. Weigand, Proc. Combust. Inst. 30 (2005) 835842.

[18] T. Poinsot, D. Veynante, S. Candel, J. Fluid Mech. 228 (1991) 561-606.

[19] T. W. Lee, D. A. Santavicca, Combust. Sci. Technol. 90 (1993) 211-229.

[20] J. F. Driscoll, D. J. Sutkus, W. L. Roberts, M. E. Post, L. P. Goss, Combust. Sci. Technol. 96 (1994) 213-229.

[21] D. A. Nye, J. G. Lee, T. W. Lee, D. A. Santavicca, Combust. Flame 105 (1996) 167-179.

[22] S. Candel, T. Poinsot, Combust. Sci. Technol. 70 (1990) 1-15.

[23] Y. Ju, H. Guo, K. Maruta, F. Liu, J. Fluid Mech. 342 (1997) 315-334.

[24] F. N. Egolfopoulos, Proc. Combust. Inst. 25 (1994) 1365-1373.

[25] C. J. Mueller, J. F. Driscoll, D. L. Reuss, M. C. Drake, Proc. Combust. Inst. 26 (1996) 347-355.

[26] T. M. Brown, R. W. Pitz, C. J. Sung, Proc. Combust. Inst. 27 (1998) $703-$ 710 .

[27] R. Sadanandan, M. Stöhr, W. Meier, Appl. Phys. B 90 (2008) 609-618. 